Meta

Journal des tradlucteurs

Translators' Journal

\title{
Pourquoi une formation à l'interprétation de conférence LSF (langue des signes française) en français et de français en LSF ?
}

\section{Philippe Séro-Guillaume}

Volume 42, numéro 3, septembre 1997

L’interprétation en langues des signes

URI : https://id.erudit.org/iderudit/002985ar

DOI : https://doi.org/10.7202/002985ar

Aller au sommaire du numéro

Éditeur(s)

Les Presses de l'Université de Montréal

\section{ISSN}

0026-0452 (imprimé)

1492-1421 (numérique)

Découvrir la revue

Citer cet article

Séro-Guillaume, P. (1997). Pourquoi une formation à l'interprétation de conférence LSF (langue des signes française) en français et de français en LSF ? Meta, 42(3), 521-532. https://doi.org/10.7202/002985ar
Résumé de l'article

En France, les interprètes pour sourds ont vu leur champ d'activités s'étendre considérablement : interprètes de liaison à l'origine, ils sont désormais amenés à assurer des prestations qui relèvent de l'interprétation de conférence. C'est pour répondre à ces besoins nouveaux que l'ESIT a mis en place en 1992 une formation de haut niveau au sein de son département Interprétation. L'originalité de la LSF - ses signifiants manuels et un énoncé qui se déroule dans l'espace - ne modifie pas le processus interprétatif- interpréter un discours n'est pas traduire une langue - ni la pédagogie de $i^{\prime}$ interprétation vers ou à partir de la LSF. 


\title{
POURQUOI UNE FORMATION À L'INTERPRÉTATION DE CONFÉRENCE LSF (LANGUE DES SIGNES FRANCAISE) EN FRANÇAIS ET DE FRANÇAIS EN LSF?
}

PHILIPPE SÉRO-GUILlaUME

Université Paris III-Sorbonne Nouvelle (ESIT), Paris, France

\begin{abstract}
Résumé
En France, les interprètes pour sourds ont vu leur champ d'activités s'étendre considérablement: interprètes de liaison à l'origine, ils sont désormais amenés à assurer des prestations qui relèvent de l'interprétation de conférence. C'est pour répondre à ces besoins nouveaux que l'ESIT a mis en place en 1992 une formation de haut niveau au sein de son département Interprétation.

L'originalité de la LSF — ses signifiants manuels et un énoncé qui se déroule dans l'espace - ne modifie pas le processus interprétatif - interpréter un discours n'est pas traduire une langue - ni la pédagogie de l'interprétation vers ou à partir de la LSF.
\end{abstract}

\begin{abstract}
While early interpreters were chiefly employed as community interpreters, at present they are also called upon to work at universities and high-level scientific or political meetings. Universite Paris III therefore introduced in 1992 a program for training French Sign Language $(L S F)$ conference interpreters. The principles and methodology are the same as for vocal interpreters.

For the purpose of interpretation, gestual signs are functionally similar to vocal signs; the same maxim applies: Interpreting a speech is not the same as translating a language.
\end{abstract}

\section{POURQUOI UNE INTERPRÉTATION DE CONFÉRENCE EN LSF ?}

Jusqu'au milieu des années 70 , les interprètes en LSF pratiquaient l'interprétation de liaison. Ils étaient requis par les tribunaux ou sollicités par les sourds eux-mêmes dans tous les cas de figure où la présence de l'interprète rendait plus commode la communication et palliait une maîtrise médiocre du français chez certains sourds (entretien avec un notaire, rendez-vous dans une administration, etc.).

Créée en 1977, une première association d'interprètes avait notamment pour but de promouvoir l'insertion sociale des sourds en leur proposant des interprètes, non seulement dans les services juridiques ou administratifs, mais aussi dans d'autres contextes: organismes de santé, manifestations à caractère culturel, politique ou religieux, formations professionnelles et universitaires.

En fait, dès 1977, les besoins en matière d'interprétation de conférence en LSF et la nécessité d'une formation pour les interprètes sont clairement identifiés. L'émergence de ce nouveau type de prestation s'explique par la conjugaison de plusieurs facteurs.

Tout d'abord, on a assisté en France à l'émergence de ce que certains sourds n'hésitent pas à appeler le «sourdisme». On trouve ce néologisme dans un tract qui, à l'occasion d'élections, attirait l'attention des candidats sur la nécessité de prendre en compte l'électorat sourd. Les militants du sourdisme ont eu à cœur de participer à toutes 
les manifestations, colloques, conférences consacrés à la surdité. Pour ce faire, ils ont fait appel à des interprètes.

Quelle que soit la nature du sujet abordé, par exemple la réhabilitation prothétique ou la construction de l'identité chez l'enfant sourd, le fait est que ces conférences sont organisées le plus souvent par des entendants qui font appel à des conférenciers entendants. Ceux-ci, universitaires pour la plupart, présentent généralement leurs travaux comme s'ils s'adressaient à des homologues. Tel n'est pas le cas. Alors que dans un congrès international, tous les participants sont des spécialistes du sujet traité, le public qui fréquente les conférences consacrées à la surdité est hétérogène. Il est composé essentiellement de professionnels de la surdité, orthophonistes et enseignants éducateurs, mais aussi de parents d'enfants sourds et, depuis une quinzaine d'années, de sourds. Les professionnels de la surdité ont certes des connaissances dans des domaines aussi variés que la linguistique, la phonétique, la physiologie de l'oreille ou le développement cognitif de l'enfant, mais pas au même titre que les conférenciers des spécialistes des sujets abordés. Bien souvent, ces professionnels de la surdité font état de leur difficulté à suivre tel ou tel conférencier. Les parents d'enfants sourds et les sourds ${ }^{1}$ qui ${ }^{\prime}$ 'ont reçu aucune formation dans les matières abordées ont encore plus de difficultés à suivre ces conférences.

Les interprètes se sont trouvés confrontés d'entrée de jeu à une tâche à laquelle ils n'étaient pas préparés. Contrairement à ce qui se passait à l'occasion d'interprétations de liaison où il s'agissait de traduire des entretiens portant sur des sujets auxquels il est arrivé à chacun d'entre nous d'être confrontés, il leur fallait interpréter des orateurs s'exprimant à jet continu, et ce, sur des sujets autrement plus ardus. Le caractère ardu des sujets traités, la non-adaptation des conférenciers au public, présentèrent d'emblée des difficultés telles aux interprètes qu'elles rendaient pressante la nécessité d'une formation.

En second lieu, des dispositions légales ont joué un rôle déterminant dans l'apparition de l'interprétation de conférence. Une loi, promulguée en 1975, préconise l'intégration scolaire de tous les enfants, quelle que soit la nature de leur handicap. Bien évidemment, les établissements spécialisés dans l'accueil des enfants sourds continuent d'accueillir les enfants dont l'intégration n'est pas envisageable mais, dans le même temps, ils concourent à l'intégration de ceux qui peuvent en bénéficier. Par ailleurs, en 1991, l'instauration légale du libre choix entre une éducation strictement oraliste ou une pédagogie dite «bilingue» associant la LSF au français parlé et écrit est venue entériner une évolution amorcée dès la fin des années 70 . L'intégration dans un établissement scolaire ordinaire de jeunes sourds a entraîné la mise en place de dispositifs d'aide spécifique; il peut s'agir de la mise à disposition de notes de cours validées ${ }^{2}$, de soutien pédagogique, de tutorat, de codage en $\mathrm{LPC}^{3}$ et, pour ceux qui ont choisi l'option bilingue, de l'interprétation en LSF des cours. À l'inverse, il est rare que les travaux à l'atelier, quel que soit le type ou le niveau de formation envisagé, menuiserie ou prothèse dentaire, soient interprétés. En effet, les élèves travaillant sur un poste, l'enseignant peut s'occuper individuellemnt de chacun d'entre eux. C'est surtout l'enseignement général et théorique délivré sous forme de cours magistraux qui nécessite la présence de l'interprète. D'une manière générale et indépendamment de l'option choisie, oraliste ou bilingue, le projet éducatif pour les jeunes sourds est plus ambitieux. Les jeunes gens sourds sont plus nombreux à accéder aux études supérieures. C'est pourquoi certains d'entre eux deviennent eux-mêmes des professionnels de la surdité. Ils enseignent la LSF, deviennent éducateurs spécialisés ou professeurs de jeunes sourds, ils enseignent alors comme leurs homologues entendants toutes les matières du programme.

L'intégration scolaire et universitaire de jeunes sourds, de même que la participation croissante des adultes sourds à des conférences et à des formations continues, a conduit les interprètes à étendre considérablement leur domaine de compétence. 
LES ARGUMENTS EN FAVEUR DU RECOURS À LINTERPRÉTATION DE CONFÉRENCE EN LSF

Tous les sourds ne sont pas logés à la même enseigne en ce qui concerne la maîtrise de la langue audiovocale et les possibilités de communication orale. La surdité, selon sa date d'apparition, pré ou post-verbale, selon son degré, profonde, moyenne ou légère, peut entraîner chez ceux qui en sont atteints des effets très variés.

Certains sourds ne maîtrisent pas suffisamment le français pour communiquer de manière approfondie oralement ou par écrit avec les entendants. Ces sourds sont obligés de recourir aux services d'un interprète pour entrer en contact avec, par exemple, un service administratif. Il ne s'agit là encore, la plupart du temps, que d'interprétation de liaison.

À côté de ce premier argument contingent, un second argument, fondamental celui-ci, plaide en faveur de l'interprétation de conférence, y compris pour les sourds qui maîtrisent le français. Dans certaines situations, conférences cours ou réunions, le recours au français parlé ou écrit ne permet pas aux sourds de communiquer naturellement.

Ces derniers ont des aptitudes très diverses à la réception d'un message vocal. Certains lisent très bien sur les lèvres, d'autres au contraire n'y parviennent qu'à grand peine. La prothèse auditive n'est utile qu'à ceux qui ont des restes auditifs. En outre, il faut noter qu'elle ne restitue pas à l'oreille toutes ses capacités de discrimination, elle amplifie non seulement la voix mais aussi tous les bruits ambiants. Enfin, son efficacité diminue très vite au fur et à mesure que la source sonore s'éloigne. En général, lorsque les échanges ont lieu entre un entendant et un sourd qui maîtrise le français et que ces deux personnes ont réellement besoin de communiquer, les adaptations réciproques (milieu silencieux si nécessaire, bonne articulation de part et d'autre - chez l'entendant pour faciliter la lecture labiale par le sourd, chez ce dernier pour rendre sa voix intelligible le recours, le cas échéant, à l'écrit ou à la gestualité naturelle) et les efforts consentis de part et d'autre permettent de venir à bout des difficultés. Il en va tout autrement lorsque des sourds assistent à une conférence, à une réunion ou à un cours. En effet, lorsqu'une personne sourde même très habile à la lecture labiale essaie de déchiffrer le message à jet continu, il faut savoir que c'est au prix d'un effort exorbitant en ce sens qu'il s'exerce au détriment des mécanismes ordinaires de compréhension et «d'écoute» (mobilisation des connaissances extra-linguistiques, associations d'idées, prise de position par rapport à ce qui est dit, activité réflexive, etc.). Qui plus est, le conférencier ne fait pas toujours face à son auditoire, il peut être éloigné des personnes sourdes. Par ailleurs, au cours d'une réunion, chacun prend la parole sans crier gare et le temps que le sourd repère qui a pris la parole, ill a manqué une bonne partie du message. S'agissant de la réception du message, les difficultés sont importantes, la lecture sur les lèvres, exercice difficile en soi, devient quasiment impossible et bien peu satisfaisante. Quelles que soient les capacités des sourds en matière de lecture labiale ou de récupération prothétique, l'interprétation de français en LSF est une nécessité dans le cadre d'un cours, d'une conférence ou d'une réunion.

S'agissant de l'expression vocale, les capacités des sourds sont très variables. Certains $^{4}$, tout en maîtrisant la LSF, sont à même de s'exprimer vocalement sans effort particulier. Ils n'ont pas besoin d'interprète pour s'adresser aux entendants; par contre, lorsque ces derniers s'adressent à eux à jet continu, ils ont besoin de l'interprétation en LSF. Pour les autres ${ }^{5}$, l'expression vocale à jet continu est un exercice difficile et peu efficace, car leur voix n'est pas toujours intelligible, elle est quelquefois même incompréhensible pour un entendant non averti. Il m'a été donné à maintes reprises de constater que l'auditoire entendant, face à une personne sourde qui fait l'effort de s'exprimer vocalement, n'ose pas lui dire qu'il ne la comprend pas de peur de la froisser. On imagine sans peine l'inconfort d'une telle situation. A première vue, dans le cadre d'une réunion ou d'une conférence, le recours à l'écrit semble être la solution idéale. On peut prendre en note simultanément les interventions des entendants pour les personnes sourdes ${ }^{6}$, on pourrait 
envisager que ces dernières s'expriment par écrit si nécessaire. Pourtant, ce n'est pas la solution préconisée par les sourds qui maîtrisent le français et la LSF. En effet, le recours à l'écrit dans les situations qui viennent d'être évoquées, s'il améliore la transmission des contenus, enlève aux échanges la spontanéité et le naturel qui caractérisent l'expression orale; il ne fournit pas aux sourds les conditions nécessaires à l'exercice naturel de la «parole». À cet égard, il ne les met pas sur le même plan que les entendants. C'est pourquoi ces derniers, y compris ceux qui maîtrisent parfaitement le français, réclament, dans leur immense majorité, pour des entretiens importants ou des conférences, une interprétation simultanée en LSF plutôt qu'une prise en note simultanée. Les étudiants sourds ${ }^{7}$ réclament aussi des interprètes pour assister à leurs cours.

\section{LA FORMATION DES INTERPRÈTES DE CONFÉRENCE}

Les prestations que doivent assurer désormais les interprètes nécessitent une qualification de haut niveau que seule une formation universitaire est à même de garantir. La création, en 1993, d'une M.S.T. (Maîtrise de Science et Technique en interprétation en LSF) à l'Université de la Sorbonne Nouvelle-Paris III dans le cadre de la section interprétation de l'ESIT indique le niveau d'exigence où se situe cette formation. Les interprètes issus de cette formation doivent être en mesure de contribuer efficacement à l'insertion sociale des sourds. Au départ, un niveau élevé de connaissances linguistiques est exigé; il est évalué dans le cadre d'un examen d'admission. Le français doit être une langue cultivée dans laquelle le candidat possède une grande richesse de vocabulaire et qu'il sait manier avec précision et aisance. La LSF doit être comprise sans hésitation dans toutes ses nuances et être un moyen d'expression rapide et précis.

Par ailleurs, les candidats doivent être titulaires d'un DEUG (deux ans d'études universitaires) ou d'un diplôme équivalent, et justifier d'une très bonne connaissance de la surdité et du milieu sourd par le biais, par exemple, d'activités au sein d'associations de sourds.

\section{Cours communs aux étudiants en interprétation de langues audiovocales et de langue des signes}

Comme leurs homologues, les étudiants de la MST reçoivent une formation en deux ans. Tout au long de ces deux années, les étudiants suivent les cours magistraux (Théorie de l'interprétation, Préparation des conférences, Méthodes d'acquisition des connaissances thématiques) de la section interprétation.

\section{Cours spécifiques aux étudiants de la MST d'interprétation de conférence en LSF}

Pour répondre aux besoins exprimés par les devenus sourds, qui n'ont qu'une connaissance médiocre de la LSF, il a été mis sur pied un enseignement de la prise en note simultanée.

Les personnes devenues sourdes après avoir acquis le français conservent la faculté de s'exprimer vocalement et ne pratiquent pas la LSF. Elles réclament des preneurs de notes qualifiés qui leur restituent par écrit ce qui est dit par les entendants dans les réunions ou les conférences auxquelles elles participent. Nous avons décidé d'appeler ce truchement très particulier Prise en note simultanée afin d'éviter toute confusion avec la prise de notes d'étudiants ou d'interprètes préparant une interprétation consécutive. Cet exercice implique de la part du preneur en note une compréhension sans faille du message et une prise de distance par rapport aux mots du discours de l'orateur qui lui permettront de trouver l'expression écrite extrêmement concise qui soit la synthèse au plan du sens et du registre de langue du discours oral original. La moindre tentative de transcription inté- 
grale du discours oral se solde par un échec par «asphyxie». Il faut aussi bien distinguer cet exercice des notes de cours que prend un étudiant qui le plus souvent filtre ce qui est dit en fonction de ses connaissances et de ses besoins, et s'autorise tous les raccourcis. Cette prestation peut encore moins être assimilée à la prise de notes qu'effectue l'interprète en vue d'une interprétation consécutive. La prise de notes de ce dernier, plus encore que celle de l'étudiant, est strictement individuelle et donc inutilisable par un tiers. On pourrait définir la prise en note pour les déficients auditifs comme la reformulation synthétique d'un discours oral faite en parallèle au fur et à mesure de son déroulement. L'exercice est infiniment plus difficile qu'il n'y paraît.

Par ailleurs, d'autres cours spécifiques ont été créés. Ils portent, par exemple, sur l'histoire de la LSF, sur la communauté sourde. Des enseignants sourds assurent un perfectionnement en LSF.

\section{L'interprétation consécutive doit être maîtrisée avant d'aborder l'interprétation simultanée}

S'agissant de l'enseignement de l'interprétation proprement dite, la première annee est consacrée à l'interprétation consécutive. L'interprétation simultanée n'est abordée qu'en seconde année. En effet, comme l'indiquent Danica Seleskovitch et Marianne Lederer (1989: 43-44):

[...] on ne saurait aborder la simultanée directement si l'on veut éviter que les étudiants tombent dans les pièges que pose le contact immédiat entre deux langues. Seule une pratique approfondie du mode consécutif d'interprétation [véritable consécutive avec notes] est en mesure d'intégrer en un savoir-faire réel, transférable à l'opération simultanée, les principes dont l'utilité aura été comprise dans les premiers exercices.

Pour que les étudiants parviennent en fin de formation à produire une interprétation simultanée réussie, il faut qu'ils sachent appliquer les méthodes interprétatives pour lesquelles la pratique de la consécutive est la plus sûre des formations.

L'interprétation de conférence consécutive français/langue des signes ou langue des signes / français n'est jamais pratiquée car, contrairement à ce qui se passe dans le cas des langues audiovocales, il est possible à l'orateur de parler ou de signer (s'exprimer en langue des signes) et à l'interprète de signer ou de parler ensemble sans se gêner tout en étant parfaitement intelligibles pour le public puisque les deux langues utilisent des modalités différentes, gestuelle-visuelle et audio-vocale. Cependant, l'enseignement de l'interprétation consécutive est absolument nécessaire pour que les étudiants comprennent les mécanismes interprétatifs.

L'interprétation consécutive puis simultanée de LSF en français et de français en LSF est travaillée comme tous les autres couples de langues audiovocales dans le cadre de travaux dirigés par des enseignants eux-mêmes interprètes.

Le postulat des théoriciens de l'ESIT, à savoir que l'interprétation n'est pas une traduction au sens d'un passage direct d'une langue à une autre, mais consiste à établir des équivalences de sens, a donné lieu à une méthode d'enseignement de l'interprétation qui est appliquée avec succès à toutes les paires de langues. Force est de constater que l'originalité de la LSF - ses signifiants manuels et un énoncé qui se développe dans l'espace - ne modifie ni la méthode interprétative ni son enseignement. Les difficultés pratiques ne tiennent pas à la nature des langues en présence, une langue audiovocale et une langue signée, en l'occurrence le français et la LSF. Le génie propre de la LSF n'est pas en cause. Ce sont des données sociologiques et historiques qui ont un retentissement sur le développement de la LSF, l'exercice de la profession et ipso facto sur la formation à l'interprétation vers ou à partir de la LSF. 


\section{Nécessité d'adapter l'interprétation au public et au contexte}

Dans le cadre de la MST, l'accent a été mis sur l'interprétation de conférence et de cours. Toutefois, à l'issue de cette formation, les interprètes seront à même de faire face à toutes les situations et à tous les publics. Ils pourront être, le cas échéant, sollicités dans les domaines les plus variés, qu'il s'agisse d'interprétation de liaison auprès des organismes ayant à connaître des sourds ou des tribunaux. Ces contraintes particulières sont prises en compte tout au long de la formation des étudiants. Leur attention est attirée sur la nécessaire adaptation aux situations et aux publics; le type de prestation variera selon qu'il s'agit d'une interprétation au tribunal, d'une conférence ou d'une formation d'éducateurs, à l'occasion d'un cours de psychologie, par exemple.

Dans le premier cas, ils ont affaire parfois, le cas est rare mais ne peut pas être passé sous silence, à des personnes sourdes qui, pour avoir eu une scolarité lacunaire, ne possèdent à proprement parler aucune langue instituée, ni le français ni la LSF, et sont peu intégrées socialement. Dans ces circonstances bien particulières, il s'agit d'indiquer clairement aux magistrats les limites de l'interprétation. Le recours au mime, au dessin, à l'explicitation, et ce, avec l'accord et l'implication des autorités judiciaires, s'avère la seule stratégie envisageable.

Dans le second cas, à l'occasion de conférences, les interprètes peuvent être amenés à travailler pour un public hétérogène, composé de personnes sourdes qui, de par leur formation, sont à même d'avoir accès au contenu de ces conférences et de personnes beaucoup moins averties, qui souhaitent que les interprètes leur rendent accessibles les contenus abstraits des conférences. Dans ces circonstances, l'interprète devra trouver un juste équilibre.

Enfin, à l'occasion de cours, a contrario, il faut préserver la spécificité du discours original, son caractère abstrait, si tel est le cas, ne pas expliciter, ne pas vulgariser. Ce point est important car, en procédant ainsi, l'interprète permet à l'étudiant sourd d'effectuer le même travail intellectuel que son homologue entendant, d'adopter la même attitude, chercher à comprendre, questionner l'enseignant. C'est à ce dernier qu'il incombe d'expliquer telle ou telle notion et non pas à l'interprète. Procéder autrement reviendrait à faire obstacle à l'action pédagogique.

En outre, dans le cadre de cours, les interprètes doivent prendre en compte le fait que le français reste la langue d'étude, la langue écrite des lycéens et des étudiants sourds. Par le truchement de l'interprétation, ces derniers doivent avoir accès au contenu des cours, mais aussi à la terminologie et aux expressions consacrées françaises propres à chaque matière pour ne pas se trouver démunis lorsqu'ils seront amenés à lire les inscriptions du professeur au tableau, les notes validées ou les ouvrages traitant de la matière enseignée. La tâche de l'interprète en LSF va donc au-delà de la seule transmission du message. L'interprète joue un rôle non négligeable dans l'approfondissement par les lycéens et les étudiants sourds de leur connaissance du français.

L'interprète de conférence doit non seulement adapter sa prestation au public et au contexte mais aussi prendre en compte des données socio-linguistiques bien particulières s'il veut produire une interprétation pertinente.

\section{Nécessité de tenir compte des données historiques}

La LSF a été élaborée par les sourds dans les institutions où ils étaient scolarisés. Souvent, ils ont été en contact avec la LSF tardivement, en tout cas à un âge, quatre, cinq ou six ans, voire plus tard, où leurs homologues entendants parlaient déjà depuis longtemps. La LSF n'est que très rarement transmise aux enfants sourds ${ }^{8}$ par leurs parents. Elle n'est enseignée de manière systématique que depuis peu ${ }^{9}$ et dans quelques établissements seulement. La forme syntaxique et rhétorique du discours en LSF, au-delà de la singularité 
de ses signifiants manuels, obéit à des schémas déterminés par l'économie du discours parlé. Les éléments paralinguistiques et surtout la connivence entre les acteurs de la communication jouent un rôle essentiel. Exclue jusqu'à une date très récente de toute situation de discours didactique, la LSF se trouve sommée de prendre en charge un champ de communication exigeant des outils lexicaux et rhétoriques dont elle n'a pas pu jusqu'ici se doter. Les interprètes sollicités pour transmettre de français en LSF des cours ou des conférences sont amenés à exprimer des notions, des concepts inédits dans cette langue. Dans le même temps, ils peuvent être amenés à introduire certains termes techniques français. Cette introduction, si elle n'est pas maîtrisée, pourrait aboutir à un transcodage généralisé.

Le risque est d'autant plus grand que certains éléments de la LSF sont dérivés du français. En effet, la LSF s'est développée en symbiose avec le français. Elle ne met pas seulement en œuvre des signes manuels autonomes et originaux. En effet, un alphabet manuel permet d'épeler en cas de besoin certains mots français et donc de les introduire dans un énoncé en LSF. Certains signes intègrent une composante alphabétique. Par exemple, le signe manuel pour dire lundi comporte le $L$ du mot français. En outre, les sourds articulent sans voix, l'information est toute entière destinée à être lue sur les lèvres - - des items dérivés de mots français. L'ensemble de ces items labialisés associés aux signes manuels est commun à tous les locuteurs sourds de la LSF. À côté de cette labialisation standard, et dans une proportion qui dépend de leur degré de maîtrise du français mais aussi du sujet abordél0, les locuteurs sourds de la LSF introduisent spontanément des expressions ou des mots français dans leur discours. Pour ce faire, ils couplent généralement un signifiant labial, le ou les mots français articulés sans voix, à un ou plusieurs signifiants manuels qui en illustrent autant que faire se peut la signification. Le cas échéant, ils épèlent manuellement ce ou ces mots. Le locuteur, tout à son propos, et bien qu'il sache parfaitement ce qu'il veut dire, ne s'embarrasse pas nécessairement de considérations d'ordre sémantique. Il choisit le signe manuel qui lui vient à l'esprit, qui lui «tombe sous la main». Comme je l'ai observé, il peut, pour signifier mesure législative, signer mesure dans le sens d'évaluation suivi d'un signe évoquant la loi ${ }^{11}$. De telles pratiques ne sont pas étonnantes si l'on songe aux conditions dans lesquelles la LSF s'est développée.

Toute langue présente des variantes plus ou moins marquées selon les régions, les milieux sociaux et même la situation de communication. Contrairement aux langues de grande diffusion, la langue des signes s'est constituée et s'est transmise jusqu'à maintenant sans bénéficier des procédures de correction des parents ${ }^{12}$ et de l'apport de l'école qui délivre un enseignement systématique de la langue et de la prise de parole. Elle n'a pas non plus bénéficié des travaux des terminologues, des grammairiens, des institutions telles que les Académies. Aussi, on comprendra que les variantes individuelles ou régionales chez les locuteurs de la LSF sont encore plus diversifiées que chez les locuteurs des langues audiovocales. De plus, la symbiose avec le français n'ayant fait l'objet d'aucune réflexion ni d'aucune concertation, l'introduction de termes ou d'expressions françaises inédits ne relèvent d'aucune procédure normalisée. interprétés :

Guidés par leurs enseignants, il s'agit, en ce qui concerne la LSF, pour les étudiants

II de se familiariser avec les variantes individuelles et régionales;

II de distinguer les modalités qui caractérisent les échanges familiers de celles qui permettent de mettre en œuvre un discours distancié, plus soutenu, plus explicite et plus articulé;

II de comprendre que rien ne s'oppose à ce qu'à l'occasion de l'interprétation d'un cours, l'interprète introduise les termes propres à la matière puisque les locuteurs sourds eux-mêmes font très spontanément des emprunts au français. Dans le même temps, les étudiants doivent prendre conscience qu'eu égard aux contextes dans lesquels ils 
auront à travailler, interprétation de conférence ou de cours, ils devront se montrer autrement plus circonspects que les locuteurs lorsqu'ils introduiront un terme français dont la polysémie ${ }^{13}$ risque d'induire en erreur les sourds;

- de repérer les limites de la symbiose avec le français. Les procédés fonctionnels, le recours à une grammaire spatiale, qui président à l'énonciation en LSF, ne sont pas affectés par les emprunts au français qui restent lexicaux et n'aboutissent jamais à un transcodage généralisé. Pour se faire une idée de l'effet produit si tel était le cas, il suffit d'imaginer un discours en français transposé mot à mot de l'anglais et truffé de mots anglais épelés à toute vitesse.

Les particularités socio-linguistiques qui viennent d'être évoquées rendent plus ardue la tâche de l'interprète. Elles mobilisent toute l'attention des formateurs.

\section{L'interprétation de conférence de LSF en français}

Les observations que $\mathrm{j}$ 'ai faites me permettent d'affirmer que les personnes qui font fonction actuellement d'interprète abordent généralement avec plus d'appréhension et moins de réussite l'interprétation de LSF en français ${ }^{14}$ que de français en LSF. Ces personnes, y compris celles dont les parents sont sourds et qui ont en quelque sorte la LSF comme langue maternelle, déclarent souvent se sentir plus à l'aise de français en LSF que de LSF en français. Il faut avoir présent à l'esprit le fait que les locuteurs de la LSF ont développé leur compétence linguistique à l'occasion de dialogues. Dans le cadre de cette interaction, le connu partagé, les adaptations mutuelles facilitent largement la compréhension. Il en va tout autrement, dans le cadre d'une conférence ${ }^{15}$ par exemple, lorsqu'il s'agit de comprendre un message signé à jet continu par un locuteur inconnu traitant d'un sujet pris hors du cadre des échanges courants.

Seuls les candidats qui ont approfondi délibérément leur compétence linguistique, en assistant assidûment aux quelques conférences données en LSF, en visionnant attentivement des enregistrements de ces conférences ou d'émissions présentées en LSF, atteignent le niveau requis pour suivre avec profit les cours de la MST. Cependant, il ne faut pas négliger les difficultés qui résultent de l'ampleur des variantes régionales, des idiosyncrasies $^{16}$ et de la prise en charge de sujets inédits.

Dans le même ordre d'idées, il faut noter que bon nombre de sourds avouent ne pas comprendre aisément certains passages de l'émission télévisée hebdomadaire en langue des signes diffusée en France. Dans cette émission, sont interviewés des entendants et des sourds. Les premiers sont interprétés simultanément en LSF, les seconds sont traduits en voix off. Les interprétations vers le français, sans doute rédigées à partir des éléments fournis par les interprètes, sont dites par des comédiens. Que l'on considère l'original ou son interprétation, on constate une certaine disparité entre les discours en français et en LSF. Les premiers sont généralement plus abstraits que les seconds.

Les difficultés signalées par les sourds méritent d'être approfondies. Le «dialecte parisien» de la LSF de l'équipe qui réalise cette émission, réputé plus riche, comporteraitil des signes inconnus des sourds de province? Les interprètes doivent-ils être mis en cause (ils n'auraient pas une connaissance suffisamment idiomatique de la LSF, ce serait pour cette raison qu'ils se risquent à interpréter simultanément de français en LSF alors qu'ils ne le font pas de LSF en français à la télévision) ? Ou bien le mal ne vient-il pas de ce que certains locuteurs sourds perdent une partie de leurs moyens parce qu'ils s'expriment à la télévision? Les sujets sont-ils abordés avec suffisamment de circonspection? La disparité entre les discours français et signés est-elle le résultat d'une adaptation légitime au public sourd ou le produit d'une conception erronée de l'interprétation ${ }^{17}$ ? Les questions posées à propos de l'interprétation à la télévision ont une portée générale ${ }^{18}$. Elles n'ont pourtant 
jamais fait l'objet d'une concertation franche entre utilisateurs et interprètes. Pour approfondir le sujet, il a été décidé de créer un groupe de travail à partir d'un noyau dur regroupant les enseignants sourds assurant le cours de perfectionnement en langue des signes à l'ESIT et les interprètes diplômés de la MST. Les travaux de ce groupe fourniront aux enseignants sourds et entendants de la MST des éléments de réflexion fort utiles.

\section{La prise de notes en consécutive de LSF en français}

On comprend, au vu des questions qui se posent à propos des prestations des interprètes en activité, que les étudiants abordent avec une certaine appréhension l'interprétation de LSF en français. Et ce, d'autant plus qu'il s'agit en première année d'interprétation consécutive avec prise de notes. Cet exercice est particulièrement difficile. Il suffit de pencher un très court instant la tête sur sa feuille de papier en écrivant pour manquer un élément important du discours en langue des signes. Par ailleurs, si l'on ne regarde pas du tout sa feuille, les notes deviennent illisibles. L'étudiant doit donc profiter des pauses de l'orateur et ne jeter que des coups d'œil très brefs sur sa feuille, ne serait-ce que pour éviter que ses notes se chevauchent. Cependant, cette difficulté spécifique à la consécutive de LSF en français ne présente pas que des inconvénients. De fait, les étudiants de la MST, tout comme leurs enseignants qui participent à ces exercices - car on ne peut enseigner que ce que l'on maitrise —, ne peuvent pas prendre de notes systématiques, ils doivent se contenter du minimum, chiffres, noms propres, énumérations, termes transcodables, un mot pour un argument. Cela en soi n'est pas une mauvaise chose puisqu'ils sont obligés d'accorder à la prise de notes la place qui lui revient dans le processus interprétatif. En effet, comme l'indiquent Seleskovitch et Lederer (1986: 44-45):

Aucun «système» de prise de notes ne peut se substituer à l'écoute du sens [...] Noter pour une interprétation consécutive n'est pas encoder de l'oral en écrit [...] Les notes de l'interprète ne sont utilisables que dans les minutes qui suivent l'intervention à interpréter - une fois achevée, celle-ci est plus présente à l'esprit de l'interprète que dans les notes, qui ravivent certains souvenirs plus qu'elles ne reproduisent le discours.

L'exercice de l'interprétation consécutive de LSF en français, malgré les contraintes qui viennent d'être évoquées, est possible à condition que l'orateur s'exprime avec aisance et clarté et que, tout en restant fluide ${ }^{19}$, il adopte un débit raisonnable. Cela montre bien que l'écoute du sens, sa mémorisation et sa restitution prévalent largement sur l'encodage linguistique.

\section{L'interprétation de français en LSF}

L'interprétation de conférence et de cours en LSF ne soulève pas moins de difficultés que l'interprétation en français. S'agissant de l'interprétation de cours tout particulièrement, l'interprète doit éviter deux écueils:

In d'une part, vouloir être totalement idiomatique. L'économie du discours didactique diffère sensiblement de celle du discours «parlé» généralement à l'œuvre en LSF;

I d'autre part, sombrer dans le transcodage généralisé à partir du français. L'interprète doit mettre en œuvre des modalités propres à la LSF qui, pour avoir été élaborées par les sourds eux-mêmes, répondent ipso facto à leurs besoins en matière de communication.

Le fait que la LSF possède un lexique moins étendu que le français va renforcer, chez ceux qui s'essayent à l'interprétation de conférence en LSF, la tendance naturelle constatée par Danica Seleskovitch et Marianne Lederer (1989: 40-41) chez les étudiants. Elles relèvent que: 
Comment dire en français «wet feeding» ou «health facility»; comment dire en anglais «rétablissement de la peine de mort»? Ces questions leur viennent plus naturellement que la question qui s'inquiète du sens: de quoi s'agit-il ?; or, la réponse à cette question leur ferait comprendre puis réexprimer sans peine le sens dont il s'agit en une occurrence donnée.

La déverbalisation, naturelle mais inconsciente dans la communication unilingue, a du mal à se produire lorsque deux langues se côtoient et que les étudiants croient encore être appelés à «traduire»; lorsqu'elle fait défaut, le transcodage reprend ses droits tandis que l'interprétation perd les siens.

Danica Seleskovitch définit la déverbalisation comme «une assimilation du sens en dehors de toute référence à la forme linguistique» (1975:5). Par ailleurs, elle précise que «le sens c'est l'idée ou si l'on préfère le vouloir dire du locuteur, et chez l'auditeur. [a fortiori chez l'interprète] c'est le compris» (1986:256).

«Les objectifs de déverbalisation et d'indépendance linguistique indispensables à la réalisation d'une interprétation exacte et intelligente» posés par Seleskovitch et Lederer (1989: 41) sous-tendent la formation. Tout au long de celle-ci, l'accent est mis sur le fait que c'est uniquement la terminologie française utilisée dans une visée encyclopédique qui doit être introduite dans l'interprétation en LSF et non les mots dont l'utilisation procède du sens commun. Lorsqu'il entend «C'est logique», l'interprète ne doit pas se demander comment «dire» logique en LSF mais comprendre que le locuteur porte une appréciation positive qui, le cas échéant, aurait pu être exprimée en d'autres termes tels que conforme au bon sens, explicable, normal, naturel et qu'il s'agit de réexprimer cette appréciation positive de manière idiomatique en LSF. Les pensées, les faits, les émotions ne sont pas désignés de la même manière par des locuteurs de langues différentes. Ce que Marianne Lederer (1981a: 357) a appelé les synecdoques du discours doivent être créées par l'interprète dans l'autre langue pour que s'établisse une équivalence entre discours. Ce n'est que dans le cas où le locuteur fait expressément référence à la logique en tant que science ayant pour objet l'étude formelle des normes de la vérité qu'il se trouve confronté à un concept inédit en LSF. Dans ce cas de figure et seulement dans ce cas de figure, il devra se préoccuper d'introduire le terme, conformément aux modalités mises en œuvre par les locuteurs de la LSF et décrites précédemment. S'agissant de l'épellation, il faut procéder sans précipitation pour laisser le temps aux sourds de déchiffrer les mots inconnus. Toutefois, l'abus de cette pratique rend les énoncés indigestes. C'est pourquoi les étudiants sont entraînés à proposer spontanément des correspondants signés pour les termes qui reviennent souvent. En procédant ainsi, l'interprète identifie clairement le concept inédit, soulage l'attention des sourds et allège sa charge de travail.

Cela étant posé, il faut s'opposer au littéralisme. L'étudiant doit comprendre que l'introduction d'un correspondant signé doit toujours résulter d'un choix délibéré et non d'une incompréhension des contenus, d'un manque d'attention au sens du discours. Faute de quoi le correspondant signé peut dans le pire des cas faire obstacle à la compréhension du concept qu'il est censé représenter. Par exemple, on a vu se répandre à la suite d'interprétations erronées un signe apparenté à résumé pour signifier synthèse. S'il est vrai qu'une réunion de synthèse réunissant professeurs, orthophonistes et éducateurs à propos d'un élève se traduit par un résumé, il est important de ne pas confondre ces deux notions. La responsabilité de l'interprète est grande lorsqu'il introduit un correspondant signé ou le cas échéant donne des explications aux personnes sourdes.

Il va de soi que l'interprète ne crée pas de néologismes signés, il propose pour le temps de l'interprétation un correspondant ponctuel du terme français. On peut espérer qu'une commission ad hoc réunissant non seulement des locuteurs de la LSF, des interprètes, des terminologues mais aussi des spécialistes des domaines abordés va être créée pour recenser, valider et, en cas de besoin, créer les néologismes ${ }^{20}$. 
L'interprétation en LSF, comme toute interprétation, doit respecter un équilibre approprié entre transcodage et interprétation proprement dite. Cet équilibre ne sera trouvé par l'interprète que s'il maîtrise les langues et possède les connaissances extra-linguistiques qui lui donnent accès aux idées, au sens des énoncés.

\section{CONCLUSION}

L'interprétation de français en LSF ou de LSF en français, malgré la dissemblance des langues en présence, vérifie le postulat posé par Seleskovitch et Lederer, à savoir qu'interpréter un discours n'est pas traduire une langue. Cette dissemblance extrême ainsi que les contraintes qui s'imposent à l'interprète font que l'interprétation vers ou à partir des langues des signes constitue un poste d'observation privilégié du processus interprétatif en ce sens qu'elle permet, me semble-t-il, de délimiter avec encore plus de netteté que lorsque l'on est en présence d'une paire de langues audiovocales, la frontière entre transcodage et interprétation proprement dite.

\section{Notes}

1. La situation a évolué positivement depuis la fin des années 70. En effet, les sourds n'avaient que très exceptionnellement accès aux études supérieures auparavant.

2. Dans le cadre de l'enseignement universitaire, il s'agit de notes prises avec un soin tout particulier par des étudiants entendants, contrôlées par l'enseignant avant d'être remises aux sourds, bien évidemment après le cours.

3. Langage Parlé Complété. Il s'agit d'un enscmble de gestes effectués sur le visage qui aident à la lecture labiale en permettant de discriminer les phonèmes qui ont une image labiale proche, par exemple japon et jambon.

4. Ceux dont la surdité n'est pas trop importante ou est apparue après l'acquisition de la langue audiovocale.

5. Ceux dont la surdité est importante ou préverbale.

6. C'est la seule solution envisageable pour les personnes devenues sourdes qui ne pratiquent pas la LSF.

7. Pour ces derniers, interprétation en LSF et mise à disposition de notes de cours validées sont complémentaires. Les étudiants sourds peuvent assister à un cours par le truchement d'un interprète en LSF, mais alors que leurs homologues entendants prennent aisément des notes tout en écoutant le professeur, les étudiants sourds sont obligés de regarder constamment l'interprète. C'est pourquoi ils ne peuvent pas prendre de notes à jet continu et ont besoin qu'on leur en fournisse à la fin du cours.

8. Seuls 7 à $9 \%$ d'entre eux - ce sont les chiffres le plus souvent avancés —ont des parents sourds.

9. En France, son utilisation a été interdite dans les classes à partir de 1880 au profit de la méthode dite «oraliste». Son enseignement systématique a commencé au début des années 1990.

10. Le lexique de la LSF fait parfoís défaut. Par ailleurs, dans certaines situations solennelles, on note l'utilisation de formules stéréotypées empruntées au français. Elles sont labialisées et transcodées manuellement.

11. L'interprète doit avoir constamment présent à l'esprit que tel ou tel item peut être à proprement parler idiomatique ou peut renvoyer à un mot ou à une expression française. L'expérience, la présence d'esprit, la connaissance du «profil linguistique» du locuteur, le contexte permettent de venir à bout de telles difficultés.

12. À l'inverse de ce qui se passe dans les sociétés de tradition orale, les jeunes sourds ne bénéficient pas de l'héritage linguistique et culturel transmis par les générations précédentes. En effet, seuls 7 à $9 \%$ (ce sont les chiffres généralement avancés) des enfants sourds ont des parents sourds. Les enfants sourds apprennent la LSF de leurs homologues lorsqu'ils commencent à fréquenter les écoles de sourds.

13. Cf. mesure : évaluation ou acte officiel visant à un effet.

14. En ce qui concerne l'interprétation de conférence, les interprétations de français en LSF sont beaucoup plus nombreuses que les interprétations de LSF en français et elles le resteront toujours. Tout simplement parce que les sourds sont moins nombreux que les entendants. Toutefois, on constate que les sourds, au fur et à mesure de leur émergence dans la société, «prennent plus la parole» et interviennent de plus en plus souvent en tant que conférenciers. De toute manière, les interprétations de français en LSF et de LSF en français sont travaillées à parts égales dans la formation, car il est fondamental que les sourds soient interprétés de manière satisfaisante.

15. Comme il a été indiqué précédemment, les sourds ont accès, petit à petit, à des formations universitaires et professionnelles de haut niveau, deviennent eux-mêmes des professionnels de la surdité et, de ce fait, se retrouvent plus fréquemment désormais en position de conférenciers. 
16. Celles-ci peuvent être constatées à l'occasion de discours traitant de sujets familiers mais aussi lorsque le locuteur aborde un sujet inédit en LSF.

17. La vulgarisation par certains interprètes des discours de français en LSF et la traduction enrichie des discours des personnes sourdes sont observables aussi dans le cadre de conférences. Elles procèdent sans doute d'une démarche de nature idéologique : les sourds doivent apparaître non seulement comme les égaux des entendants mais, qui plus est, comme les homologues des entendants les plus cultivés. Délibérées ou non, ces pratiques produisent un effet de masque quant à la réalité et à la diversité socio-culturelle de la communauté sourde.

18. Cf. Séro-Guillaume (1994).

19. L'orateur, tout en s'exprimant posément, doit absolument rester fluide, sinon l'exercice se transformerait rapidement en dictée et perdrait toute valeur propédeutique.

20. D'une manière générale, il s'agit de contribuer non plus individuellement, comme l'ont fait les traducteurs lorsqu'ils ont restitué de grands textes scientifiques ou religieux dans des langues dites vulgaires à l'époque, telles que le français, le suédois, l'allemand ou l'anglais, mais collectivement au développement, à l'enrichissement de la langue des signes. Cf. Les traducteurs dans l'histoire, sous la direction de Jean Delisle et Judith Woodsworth.

\section{RÉFÉRENCES}

DELISLE, Jean et Judith WOODSWORTH (dir.) (1995): Les traducteurs dans l'histoire, Ottawa, Les Presses de l'Université d'Ottawa, Éditions UNESCO.

LAPLACE, Colette (1994): Théorie du langage et théorie de la traduction. Les concepts-clefs de trois auteurs: Kade, Coseriu, Seleskovitch, Paris, Didier Érudition.

LEDERER, Marianne (1981a) : La traduction simultanée, Paris, Minard, Lettres modernes.

LEDERER, Marianne (1981b) : «La pédagogie de l'interprétation simultanée», J. Delisle (réd.), L'enseignement de l'interprétation et de la traduction, Cahiers de traductologie 4, Ottawa, Éditions de l'Université d'Ottawa.

LEDERER, Marianne (1994) : La traduction aujourd' hui, Paris, Hachette.

SELESKOVITCH, Danica (1968) : L'interprète dans les conférences internationales, $2^{\circ}$ édition 1983, Paris, Minard, Lettres modernes.

SELESKOVITCH, Danica (1975) : Langage, langues et mémoire, Paris, Minard, Lettres modernes.

SELESKOVITCH, Danica (1981) : «L'enseignement de l'interprétation», J. Delisle (réd.), L'enseignement de l'interprétation et de la traduction, Cahiers de traductologie $\mathrm{n}^{\circ} 4$, Ottawa, Éditions de l'Université d'Ottawa.

SELESKOVITCH, Danica et Marianne LEDERER (1986): Interpréter pour traduire, $2^{\mathrm{e}}$ édition, Paris, Didier Érudition.

SELESKOVITCH, Danica et Marianne LEDERER (1989) : Pédagogie raisonnée de l'interprétation, Paris, Didier Érudition.

SÉRO-GUILLAUME, Philippe (1990): «Le sens, le geste et le verbe», Études traductologiques en hommage à D. Seleskovitch, Paris, Minard, Lettres modernes.

SÉRO-GUILLAUME, Philippe (1994) : L'interprétation en langues des signes française, thèse de doctorat, Paris, ESIT de l'Université de la Sorbonne Nouvelle, Paris III.

SÉRO-GUILLAUME, Philippe (1996) : «La formation à l'interprétation en langue des signes française à l'ESIT», XIVth World Congress of the Fédération Internationale des Traducteurs, Proceedings Volume 1. 